\title{
Continuous damage fiber bundle model for strongly disordered materials
}

\author{
Frank Raischel, ${ }^{1, *}$ Ferenc Kun, ${ }^{2}$ and Hans J. Herrmann ${ }^{1,3}$ \\ ${ }^{1}$ ICP, University of Stuttgart, Pfaffenwaldring 27, D-70569 Stuttgart, Germany \\ ${ }^{2}$ Department of Theoretical Physics, University of Debrecen, P. O. Box:5, H-4010 Debrecen, Hungary \\ ${ }^{3}$ Computational Physics, Institute for Building Materials, HIF E12, ETH Hönggerberg, CH-8093 Zürich, Switzerland
}

(Received 11 June 2007; published 2 April 2008)

\begin{abstract}
We present an extension of the continuous damage fiber bundle model to describe the gradual degradation of highly heterogeneous materials under an increasing external load. The breaking of a fiber in the model is preceded by a sequence of partial failure events occurring at random threshold values. In order to capture the subsequent propagation and arrest of cracks, furthermore, the disorder of the number of degradation steps of material constituents, the failure thresholds of single fibers, are sorted into ascending order and their total number is a Poissonian distributed random variable over the fibers. Analytical and numerical calculations showed that the failure process of the system is governed by extreme value statistics, which has a substantial effect on the macroscopic constitutive behavior and on the microscopic bursting activity as well.
\end{abstract}

DOI: 10.1103/PhysRevE.77.046102

PACS number(s): 46.50.+a, 62.20.M-, 81.40.Np

\section{INTRODUCTION}

Materials with a highly disordered microstructure exhibit a variety of constitutive characteristics when subjected to an increasing external load-from perfectly brittle to perfectly plastic as well as strain hardening and softening [1,2]. Experiments have revealed that even if the constituents are brittle, the macroscopic behavior shows these variations, especially if the internal structure of the specimen has a hierarchy of length scales. A possible explanation of this observation is the gradual activation of failure mechanisms relevant at different length scales of the materials' microstructure [3]. This interaction of microstructure and failure mechanism is typical for fiber reinforced composites, where fibers of a few micrometers diameter are embedded in a matrix material [4]. The same geometrical arrangement is found on a larger scale when pieces of timber are glued together to form high strength wood structures [5]. In such materials the microscopic origin of gradual degradation is the accumulation of damage in fibers and timber pieces due to the growing population of microcracks [6,7], furthermore, the growth and arrest of larger cracks spanning over several length scales of the specimen [8-10].

Fiber bundle models (FBMs) are one of the most adequate approaches to understand the fracture of heterogeneous materials [11-14]. Recently a continuous damage fiber bundle model (CDFBM) has been introduced where the stiffness of fibers is reduced in subsequent failure events representing the gradual degradation mechanism of the material $[3,15,16]$. Recent experiments revealed that due to the highly heterogeneous microstructure of faults, defects (like the knotholes of wood [17]), and also particularly failure resistant spots, the maximum number of failure of composites' constituents show strong variations inside samples, which has also an important effect on the evolution and propagation of cracks. Since the stress concentration around the tip of the crack increases with the crack length, cracks that grow larger than

\footnotetext{
*raischel@icp.uni-stuttgart.de
}

a certain critical size are unstable [1]. However, cracks can also become arrested at strong locations inside the material, where the stress concentration around the growing crack demands an increasing strength of subsequent arrest locations $[9,10,18]$. This phenomenon therefore requires the knowledge of the distribution of the strongest points in the sample, an issue that is related to order statistics [19,20].

In this paper we propose an extension of CDFBM in order to capture the experimentally relevant failure mechanisms of composite materials discussed above. We consider a bundle of parallel fibers which undergo a sequence of failure events when the local load on them exceeds the corresponding threshold values. The maximum number of allowed failures is a Poisson distributed random variable in the model which accounts for the disorder of degradation steps of fibers in composites. Since growing cracks sweep over locations of increasing strength, in the model the breaking thresholds of consecutive failure events of single fibers are sorted in ascending order. We explore the complexity of the model by analytical and numerical calculations and show that the extreme value statistics of failure thresholds has a substantial effect on the fracture process. Contrary to the conventional CDFBM, the macroscopic response of the system does not have a plastic plateau, but instead strain hardening occurs. Computer simulations revealed that on the microlevel the burst size distribution follows a power law with a crossover of the exponent from $5 / 2$ to $3 / 2$ when changing the parameters of the model. Beyond the general interest, our model can in particular be applied to describe the stability of large scale composite wood structures. Following the catastrophic collapse of several buildings composed of such structures in the winter of 2006/2007, see, e.g., [21], this issue has received growing interest in the recent past.

\section{RANDOMLY DISTRIBUTED FAILURE NUMBERS}

As a model we employ a continuous damage fiber bundle model (CDFBM), in which the damage law of the classical dry fiber bundle model (DFBM) is supplemented by a gradual degradation of fiber strength in the sequence of fail- 
ure events [3,15]. It was shown in Ref. [15] that for certain choices of the model parameters a variety of experimental situations can be recovered, i.e., either strain hardening or plasticity can occur. On the microscale, the size distribution of avalanche events shows a power law behavior, but the exponent is different from the ordinary FBM [22], and for certain choices of parameters an exponential cutoff appears [15].

The CDFBM is constructed as follows: the bundle consists of $N$ parallel fibers on a square lattice with identical Young-modulus $E$ and random failure thresholds $\sigma_{t h}^{i}, i$ $=1, \ldots, N$ with a probability density $p$ and distribution function $P$. Under loading, the fibers behave linearly elastic until they reach their respective points of failure and break in a brittle manner, i.e., as soon as the load on a fiber exceeds its breaking threshold $\sigma_{t h}^{i}$, the fiber will fail. If the external strain is kept fixed (strain-controlled loading), then the fibers break one by one in the order of their breaking thresholds, and the full constitutive curve is explored. Under stress controlled loading, however, the load on a breaking fiber is redistributed on the remaining intact ones, where it can trigger subsequent avalanches of fiber breakings [23]. At the maximum of the constitutive curve, a catastrophic avalanche then results in failure of the entire system [22,24].

The failure law of the DFBM is modified in the CDFBM by assuming that at the failure point the Young modulus of the fiber is reduced by a factor $a$, where $0 \leq a<1$; consequently the stiffness of the fiber after failure is $a E$. The loading of the fiber will then resume in a linear manner with the reduced stiffness until the next breaking threshold is reached. The parameter $k_{\max }$ determines the maximum number of failures allowed for a single fiber. The damage threshold $\sigma_{t h}^{i}$ can either be kept constant for all the breakings (quenched disorder) or new failure thresholds of the same distribution can be chosen (annealed disorder) after each instant of failure, which can model a microscopic rearrangement of the material after failure, cf. Figs. 1(a) and 1(b) [25-29].

It can be assumed that in an actual experimental situation, the number of times that a constituent of the material can break is an independent realization of an integer random variable. A prime example is the fracture of wood, specifically of glued timber [5,30], where only a few large defects and the finite number of glued joints determine the extreme value statistics that governs the propagation and arrest of cracks. This fact can be incorporated by modeling $k_{\max }$ as a random number, which is governed by a Poissonian distribution

$$
n_{\kappa}\left(k_{\max }\right)=\frac{\kappa^{k_{\max }} e^{-\kappa}}{k_{\max } !} .
$$

A new parameter then enters the model, which is the mean value of $k_{\max }, \kappa=\left\langle k_{\max }\right\rangle$. With this prescription, the macroscopic response of the system can be expressed as

$$
\sigma=\varepsilon \sum_{k_{\max }=0}^{\infty} \frac{\kappa^{k_{\max }} e^{-\kappa}}{k_{\max } !}\left[\sum_{k=0}^{k_{\max }} a^{k} P_{k}(\varepsilon)\right],
$$

i.e., the Poissonian distribution is convoluted with the degradation term obtained for the constitutive behavior in the con-
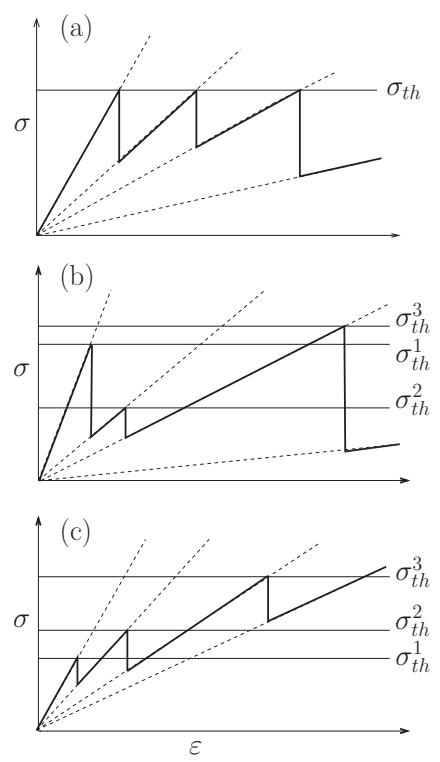

FIG. 1. Damage law for a single fiber for the cases of (a) quenched and (b) annealed disorder, cf. [3], and (c) for the model with sorted thresholds discussed in Sec. III. In all cases, after breaking of a fiber upon reaching a failure threshold $\sigma_{t h}^{i}$, loading is resumed with a stiffness that is reduced by a factor $a$.

tinuous damage model [3]. In the case of quenched disorder for the failure thresholds, the probabilities $P_{k}(\varepsilon)$ that at a given deformation $\varepsilon$ a randomly chosen fiber has failed exactly $k$ times is

$$
P_{k}(\varepsilon)= \begin{cases}1-P(\varepsilon), & k=0 \\ P\left(a^{k-1} \varepsilon\right)-P\left(a^{k} \varepsilon\right), & 1 \leq k \leq k_{\max } . \\ P\left(a^{k_{\max }-1} \varepsilon\right), & k=k_{\max }\end{cases}
$$

For the following discussion, the damage thresholds will be drawn exclusively from a Weibull distribution with $\lambda=1, m$ $=2$, unless otherwise mentioned.

We can also apply this model to the case of annealed disorder, where

$$
P_{k}(\varepsilon)=\left\{\begin{array}{ll}
{\left[1-P\left(a^{k} \varepsilon\right)\right] \prod_{j=0}^{k-1} P\left(a^{j} \varepsilon\right),} & 0 \leq k \leq k_{\max }-1 \\
\prod_{j=0}^{k_{\max }-1} P\left(a^{j} \varepsilon\right), & k=k_{\max }
\end{array},\right.
$$

however, we restrict this discussion to the quenched disorder case. In Sec. III, a different concept will be introduced which to a certain extent describes a third alternative to the cases of quenched and annealed disorder.

In the analytical solution for the constitutive behavior, Eq. (2), two physically strongly distinct cases can be realized by appropriate choices of the summation limit of the innermost, bracketed term: if the summation extends from zero to $k_{\max }$, as indicated, the fibers will retain a residual stiffness after the limiting case of $k_{\max }$ failures, i.e., hardening of the fiber bundle occurs in the limit of large $\varepsilon$, and the asymptotic behavior of the bundle is described by 


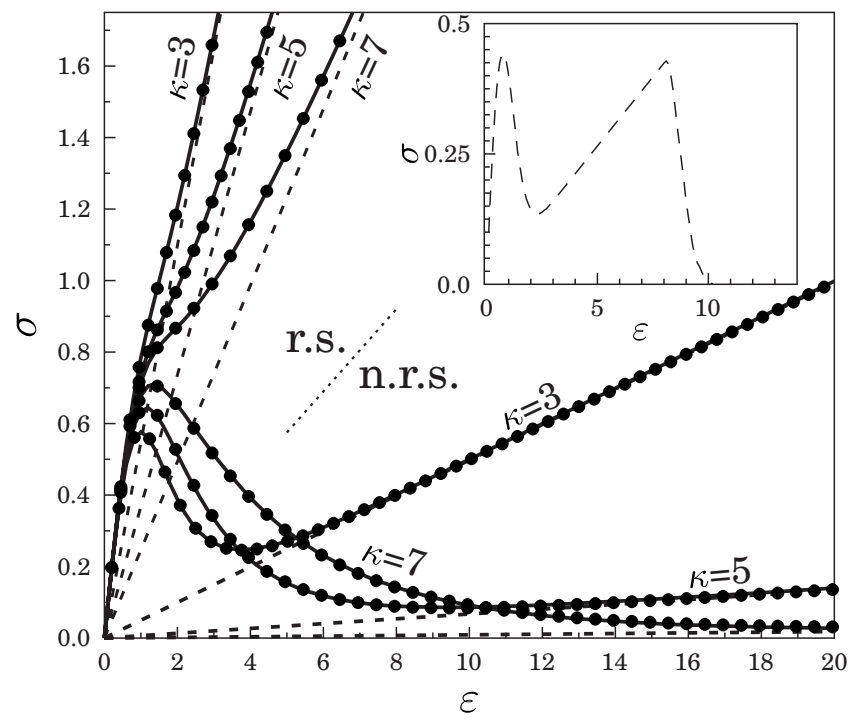

FIG. 2. Constitutive behavior for a fiber bundle with quenched disorder and Poisson distributed $k_{\max }$ for three values of $\kappa$, with (r.s.) and without (n.r.s.) residual stiffness. Symbols: analytical solution, Eq. (2); solid lines: simulation results. The dashed lines show the asymptotic behavior in the presence of residual stiffness, Eq. (5), and without, Eq. (7). Inset: mixed fiber bundle with a second shifted Weibull distribution centered around $\hat{\lambda} \approx 9$.

$$
\sigma_{\text {asympt. }}=\varepsilon \sum_{k_{\max }=0}^{\infty} \frac{\kappa^{k_{\max }} e^{-\kappa}}{k_{\max } !} a^{k_{\max }}=\varepsilon e^{-\kappa(1-a)} .
$$

Figure 2 demonstrates the perfect agreement between the analytical solution, Eq. (2), and a strain controlled numerical simulation, where the asymptotic linear behavior of Eq. (5) is recovered. In order to model the failure of materials, however, the failure law has to be slightly modified: after $k^{*}$ $=k_{\max }-1$ failures, the load on a fiber must be set to zero, and the constitutive behavior changes to

$$
\sigma=\varepsilon \sum_{k_{\max }=0}^{\infty} \frac{\kappa^{k} \max e^{-\kappa}}{k_{\max } !}\left[\sum_{k=0}^{k^{*}} a^{k} P_{k}(\varepsilon)\right]
$$

Again, in this case the constitutive curves displayed in Fig. 2 show an excellent agreement between the analytical solution, Eq. (6), and the simulation data. At first glance, the hardening behavior that emerges after the main course of loading may appear to contradict the fact that residual stiffness is not explicitly taken into account and the terms $P_{k}(\varepsilon)$ with $k$ $=k_{\max }$ are excluded in the failure law, Eq. (6); this regime is dominated by the fibers with $k_{\max }=0$, i.e., fibers that never break, and since the expectation value of these fibers is $n_{\kappa}\left(k_{\max }=0\right)=e^{-\kappa}$, the asymptotic behavior even in the case without an explicit residual stiffness reads

$$
\sigma_{\text {asympt }}=\varepsilon e^{-\kappa}
$$

Apparently, the dominance of the fibers with $k_{\max }=0$ diminishes with increasing $\kappa$, and at least for this choice of the

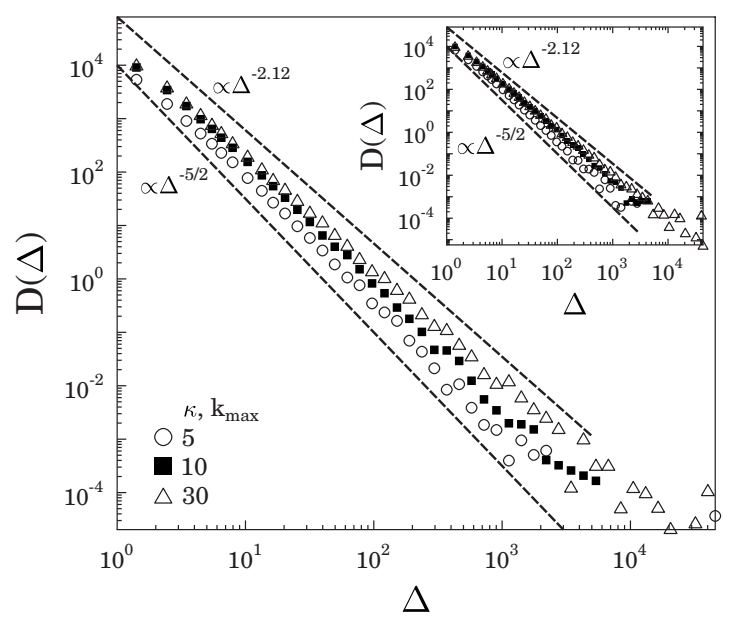

FIG. 3. Avalanche size distribution for a fiber bundle of size $L$ $=201$ with Poisson distributed $k_{\max }$ for different values of $\kappa$. Inset: Avalanche size distribution for a fiber bundle of size $L=201$ with fixed $k_{\max }$, where the $k_{\max }$ values shown correspond to the $\kappa$ values of the main plot.

disorder distribution, under stress controlled loading the hardening regime cannot be accessed, i.e., all fibers break before traversing the local minima of the slope.

In a real material unbreakable fibers are not present, but the hardening behavior of the $k_{\max }=0$ fibers can account for a second class of fibers with much higher failure thresholds than the majority fibers. Although the behavior of Eq. (5) cannot be observed asymptotically, the Poissonian term with $k_{\max }=0$ gives a reasonable approximation of this situation. In the inset of Fig. 2 we show the constitutive behavior of a mixed FBM in the spirit of [31], where a second species of fibers with a relative weight of $1 / e^{3}$ is introduced. This species has a narrow Weibull-like strength distribution and is shifted to an average value of $\approx 9$. These high strength fibers "survive" the failure of the majority species at low strains, and apparently - for intermediate strains - this second family of fibers causes a constitutive behavior that is equivalent to the continuous damage model with $\kappa=3$.

Concerning the avalanche size distribution, this model reproduces the behavior of the case of fixed $k_{\max }$, which has been discussed in [15]. There, it was found that for larger values of $a$, corresponding to $a>0.3$ for the Weibull distribution, the distribution can be fitted to a power law, the exponent of which also depends on $k_{\max }$. For small values of $k_{\text {max }}$, the usual mean field behavior with an exponent $-5 / 2$ is obtained [22], whereas for larger values of $k_{\max }$ a smaller exponent $\approx 2.12$ appears. Examining the effect of the Poissonian term with a choice of $\kappa=\left\langle k_{\max }\right\rangle$ corresponding to the ordinary CDFBM, we find in Fig. 3 a quantitative agreement between the two models, i.e., the Poissonian term causes no visible change to the avalanche statistics, and even the crossover between the two power law exponents is recovered.

\section{SORTED FAILURE THRESHOLDS}

In highly disordered materials subjected to an increasing external load, cracks nucleate in the early stages of loading at 
the weakest locations in a spatially random manner. As the load increases, simultaneously to the nucleation of new microcracks the existing cracks propagate and become unstable. Advancing cracks can be arrested by high strength locations of the material. Before macroscopic failure occurs, advancing cracks can undergo several activation and arrest events. Since stress concentration at the crack tip increases as the crack becomes longer [1], arresting can only be realized by local material elements of increasing strength. These growth and arrest events result in a gradual degradation of the macroscopic sample strength.

In order to provide a more realistic representation of this gradual degradation process sweeping through material elements in the increasing order of their local strength, we further extend the CDFBM by sorting the activation thresholds into increasing order. Figure 1(c) provides a graphical illustration of the sorting and the ensuing damage law for a single fiber. It is important to emphasize that from a physical point of view this case is a mixture of the annealed and quenched disorder cases discussed previously [3]. On the one hand, the sorted model bears resemblance to annealed disorder, since the consecutive thresholds are different from each other; on the other hand, it could also be classified as quenched as the thresholds are fixed in advance. Sorting of a series of random numbers imposes a correlation between these numbers, and we will have to resort to a mathematical theorem from the field of order statistics in order to obtain a complete understanding of the failure mechanism. It should be stressed that in the following discussion we will consider $k_{\max }$ to assume a fixed value, although the addition of the randomly distributed $k_{\max }$ can be trivially incorporated. Here a single fiber-seen as a meta element representing smaller constituents-models the progress of a crack, which does not proceed continuously but comes to a halt at certain values of the fibers' strain $\varepsilon$. We may apply the damage law of the CDFBM, but impose the additional condition that the load on the fiber at subsequent instants of the arrest should increase. Hence we can draw the failure thresholds from a random distribution, for which we will use again the Weibull distribution, and store them in sorted order. As in the previous discussion, the Weibull distribution employed will have the parameters $\lambda$ $=1$ and $m=2$.

\section{A. Macroscopic response}

As mentioned above, bringing an array of $n$ random numbers in sorted order necessarily invokes correlations between them, and the distribution of the random variant at the $i$ th position is not governed by the PDF of the unsorted random numbers anymore.

From order statistics, the following results pertaining to a sequence of random numbers $X_{1}, \ldots, X_{n}$ drawn from a CDF $F(x)$ and density $f(x)$ are known [20]: if the $X_{i}$ are brought into increasing order, then the numbers $X_{(i)} \leq \cdots \leq X_{(n)}$ are called the order statistics of the $X_{i}$, which have the joint density

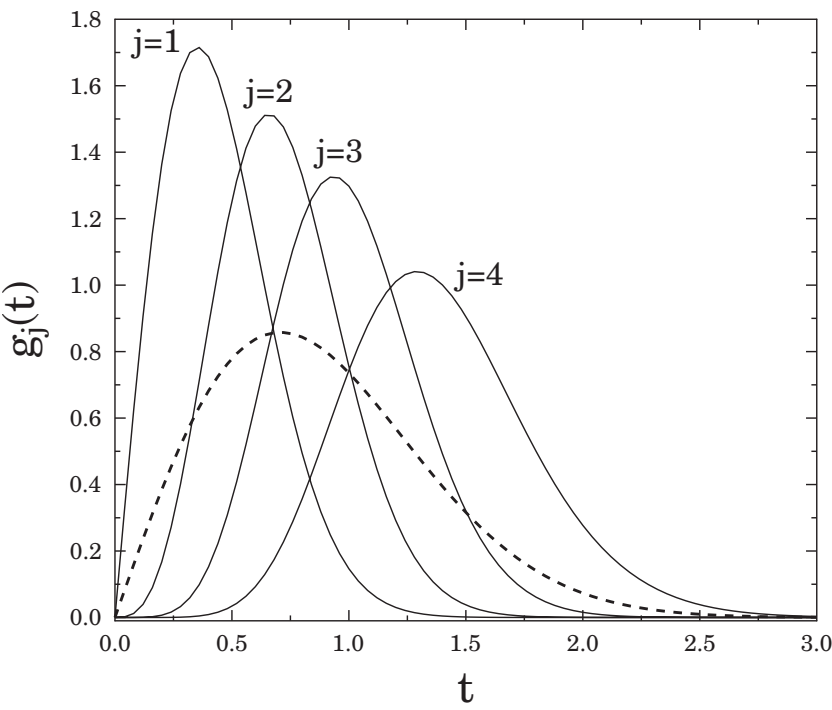

FIG. 4. Marginal densities for the order statistics of a Weibull distribution with $\lambda=1, m=2$, for the respective values $j=1, \ldots, n$ $=4$ (solid lines), together with the underlying PDF (dashed line); $\mathrm{cf}$. Eq. (9).

$$
\begin{aligned}
& g\left(x_{(1)}, \ldots, x_{(n)}\right) \\
& \quad= \begin{cases}n ! \prod_{i=1}^{n} f\left(x_{(i)}\right), & -\infty<x_{(1)}<\cdots<x_{(n)}<\infty \\
0, & \text { elsewhere }\end{cases}
\end{aligned}
$$

The marginal density for the $j$ th order statistics $X_{(j)}, 1 \leq j$ $\leq n$ is

$$
\begin{gathered}
g_{(j)}(t)=\frac{n !}{(j-1) !(n-j) !}[F(t)]^{j-1}[1-F(t)]^{n-j} f(t), \\
-\infty<t<\infty .
\end{gathered}
$$

The marginal statistics Eq. (9) is therefore the adequate replacement of the PDF, i.e., the distribution function for the random number at the $j$ th position, if $n$ random numbers have been drawn. In order to illustrate this result, in Fig. 4 the marginal statistics for the $j$ th random number, $1 \leq j \leq n$ $=4$, together with the underlying Weibull distribution with $\lambda=1, m=2$ is shown. It is apparent from Fig. 4 that with increasing $j$, the marginal statistics share the peaked characteristics of the underlying PDF, and that the position of the maxima reflects the sorting. Also, the marginal statistics become wider with increasing $j$, although this effect is not too pronounced.

Having found an analytical expression for the marginal statistics, the constitutive behavior of this model can be expressed in a closed form. In analogy to the annealed case of the CDFBM, we denote by $P_{k}(\varepsilon)$ the probability that a fiber has failed exactly $k$ times at a strain $\varepsilon$ : 


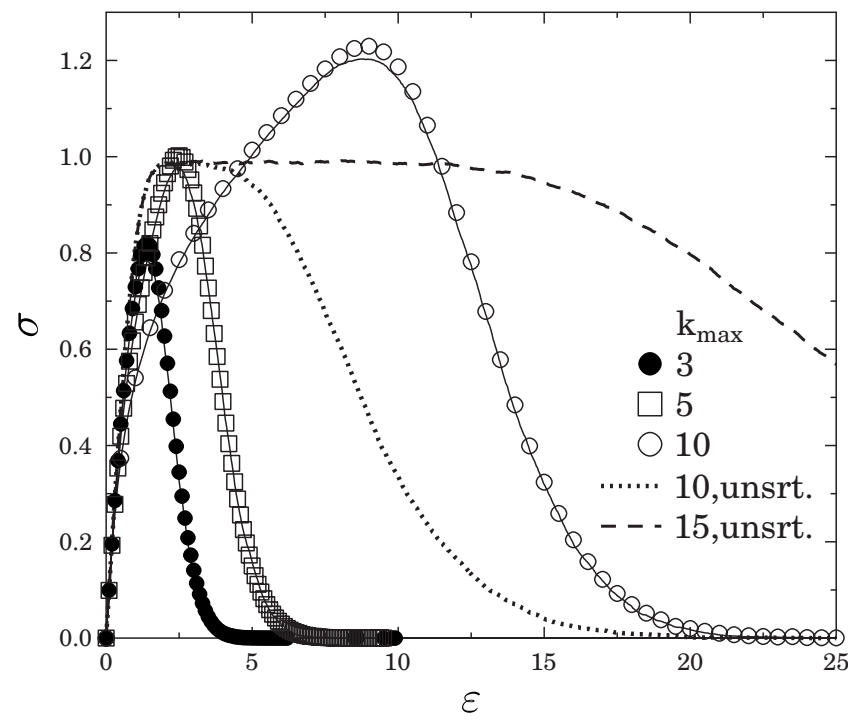

FIG. 5. Constitutive curves for the model with sorted random thresholds, without residual stiffness. Solid lines: simulation results; symbols: analytical solution Eq. (12); dashed and dotted lines: simulations for the conventional CDFBM without sorting, shown for comparison. The failure thresholds are drawn from a Weibull distribution with $\lambda=1, m=2$.

$$
\begin{aligned}
P_{k}(\varepsilon) & \left\{\begin{array}{ll}
{\left[1-G_{(k+1)}\left(a^{k} \varepsilon\right)\right] \prod_{j=0}^{k-1} G_{(j+1)}\left(a^{j} \varepsilon\right),} & 0 \leq k \leq k_{\max }-1 \\
\prod_{j=0}^{k_{\max }-1} G_{(j+1)}\left(a^{j} \varepsilon\right), & k=k_{\max }
\end{array},\right.
\end{aligned}
$$

where

$$
G_{(j)}(x)=\int_{0}^{x} g_{j}(t) d t
$$

is the integral associated with the marginal statistics $g_{(j)}(t)$, corresponding to the $\mathrm{CDF}$ of unordered random numbers. The second case in Eq. (10) with $k=k_{\max }$ corresponds to the residual stiffness of the bundle. With this result the analytic solution for the constitutive behavior reads

$$
\sigma(\varepsilon)=\sum_{k=0}^{k_{\max }-1} a^{k} \varepsilon\left[1-G_{(k+1)}\left(a^{k} \varepsilon\right)\right] \prod_{j=0}^{k-1} G_{(j+1)}\left(a^{j} \varepsilon\right),
$$

if the hardening term is skipped in order to account for material failure.

It should be noted, though, that in the formula for the $k$ th failure probability $P_{k}(\varepsilon)$, Eq. (10), the integral Eq. (11) appears, which cannot in general be solved analytically due to the structure of the integrand, Eq. (9). In order to obtain the constitutive behavior the integral Eq. (11) has to be evaluated numerically. In Fig. 5, the stress-strain curves obtained in this way are plotted for three values of $k_{\max }$, where an excellent agreement between the numerical and analytical results,

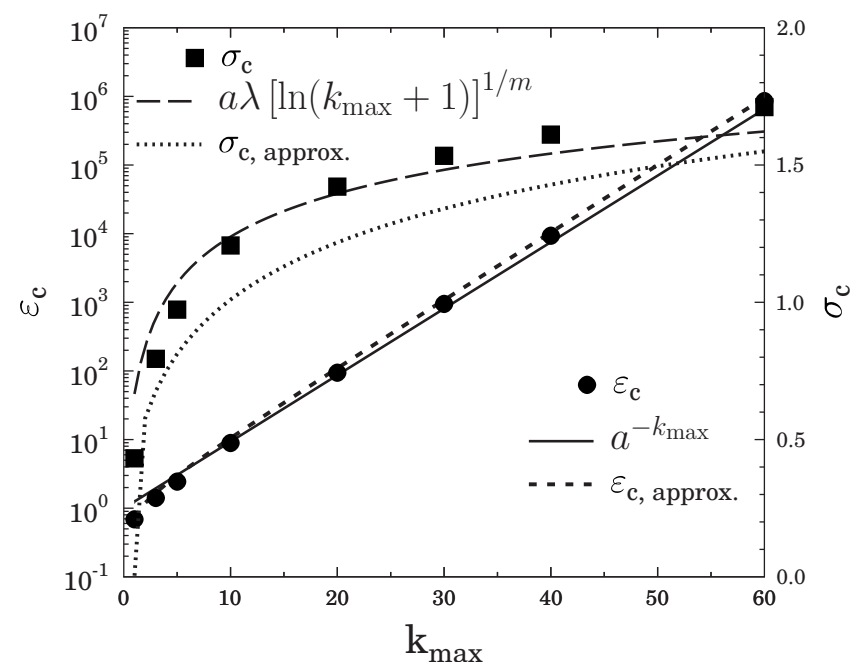

FIG. 6. The critical stress $\sigma_{c}$ and strain $\varepsilon_{c}$ for a bundle with sorted random thresholds from a Weibull distribution with $\lambda=1$, $m=2$ and without residual stiffness as a function of the maximum number $k_{\max }$ of failures. The symbols represent simulation results, whereas the lines display evaluations of Eqs. (13) and (14) and numerically obtained maxima of Eq. (15) with the setting $k=k_{\max }$, respectively.

Eq. (12), can be observed. It can be seen in Fig. 5 that contrary to the case of unsorted thresholds [15]—-whether quenched or annealed disorder is of no importance-that the constitutive curve does not develop a plateau; it always increases monotonically and has a quadratic maximum where macroscopic failure occurs. As a consequence of extreme value statistics, with growing $k_{\max }$ the critical stress $\sigma_{c}$ and strain $\varepsilon_{c}$ increase, indicating a higher macroscopic load bearing capacity. It can be seen from the general expression of the constitutive curve, Eq. (12), that the macroscopic failure of the system is mainly controlled by the largest thresholds whose distribution can be obtained from Eq. (9), setting $j$ $=k_{\max }$. Analyzing the constitutive behavior of the system considering only the largest thresholds, $j=k_{\max }$, yields

$$
\sigma_{c} \approx a \lambda\left[\ln \left(k_{\max }+1\right)\right]^{1 / m}
$$

and

$$
\varepsilon_{c} \approx a^{-k_{\max }},
$$

for the failure stress and strain, respectively, assuming Weibull distributed failure thresholds with parameters $\lambda, m$. Equation (13) implies that the sorted CDFBM does not have a plastic limit as in the conventional CDFBM, i.e., no plateau of the $\sigma(\varepsilon)$ emerges. Instead, the strength of the bundle is an asymptotically increasing function of $k_{\max }$, namely, $\sigma_{c}$ increases logarithmically whereas $\varepsilon_{c}$ increases exponentially with $k_{\max }$. This is illustrated in Fig. 6, where the values $\sigma_{c}$ and $\varepsilon_{c}$ obtained by computer simulations are compared to the analytical results, Eqs. (13) and (14).

For very high values of $k_{\max }$, a distinguished regime of oscillations appears in the constitutive curves; see Fig. 7. This plot shows the constitutive behavior in a stress controlled simulation with $k_{\max }=60$, together with the avalanche 


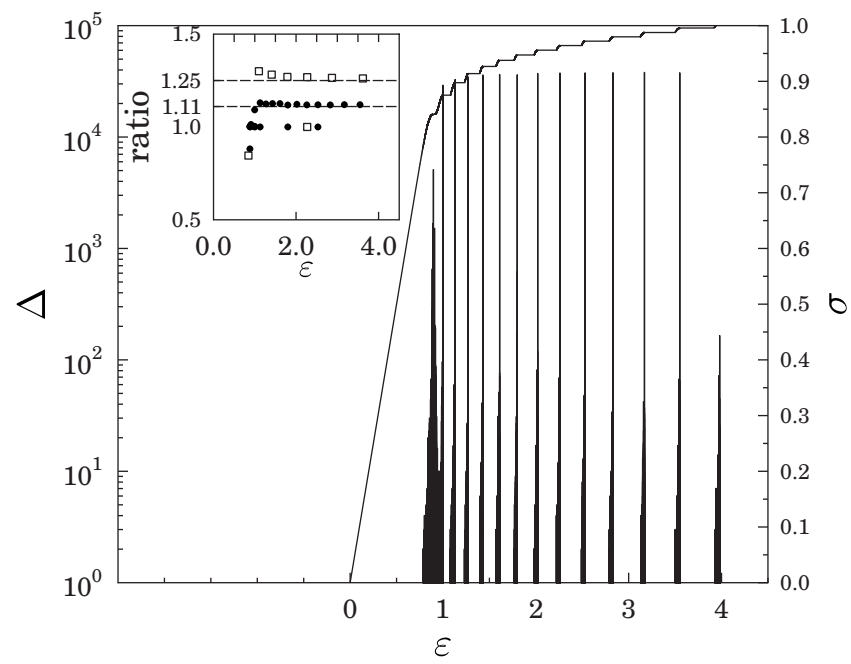

FIG. 7. Constitutive curve (solid line) and avalanche sizes without residual stiffness as a function of strain $\varepsilon$ from a simulation with $k_{\max }=15, m=4$ and $\lambda=0.9$. Inset: ratio $\varepsilon_{i+1} / \varepsilon_{i}$ for subsequent bursts with $\Delta_{i}>200$, for the values $a=0.8$ (unfilled squares) and $a=0.9$ (filled circles), where the lines indicate the respective values of $1 / a$.

sizes $\Delta$ recorded at each loading step. Apparently, the constitutive curve displays a large amount of oscillations with horizontal plateaus, which coincide with large scale bursts of breaking events. The position of the peaks suggests a regularity of some kind. In order to quantify this regularity of the peak events, the inset carries information about the ratios $\varepsilon_{i+1} / \varepsilon_{i}$ of subsequent failure events $\Delta$ with $\Delta_{i}>2000$. Obviously, this ratio assumes a constant value of $\approx 1 / a$ after a brief onset period, where $a=0.8$ is the load reduction parameter used in all the simulations presented in this discussion, and a comparison with the case of $a=0.7$ is presented in order to confirm the influence of the load reduction parameter. It should also be noted that the envelope of the constitutive curve remains monotonically increasing. In order to analyze this oscillation phenomenon an investigation on the evolution of the breakdown process is necessary.

In fact, an analytical argument can be made about the origin of these oscillations, and their position relative to the state of loading $\varepsilon$. In the constitutive formula, Eq. (12), there appears a product of integral marginal distributions $G_{(j+1)}\left(a^{j} \varepsilon\right)$, where $0 \leq j \leq k-1$, and $0 \leq k \leq k_{\max }-1$. From the structure of Eq. (9), and if a Weibull distribution with $m=2$, $\lambda=1$ is assumed as usual, it can be understood that the respective derivatives $g_{(j+1)}(x)$ take on their maximum value even for very large $j, n$, and $j \leqslant n$ at a numerical value of the argument $x=\mathcal{O}(1)$. Therefore the $g_{(j+1)}$ for large values of $j$ possess well defined peaks at very large values of $\varepsilon$, where $a^{j} \varepsilon \approx 1$. The positions of these peaks become strongly separated for subsequent indices $j, j+1$ if $j=\mathcal{O}(n)$ and $n$ is large. Consequently, in Eq. (12) the product of the integral quantities $G_{(j+1)}\left(a^{j} \varepsilon\right)$ can be replaced by the largest factor $G_{(k)}\left(a^{k-1} \varepsilon\right)$ for relatively large $k$, and together with the leading term $\varepsilon\left[1-G_{(k+1)}\left(a^{k} \varepsilon\right)\right]$ a peak structure is formed. It was confirmed numerically for $k_{\max }=60$ and $k \gtrsim 30$ that the positions $\varepsilon$ of these maxima, which are defined through the function

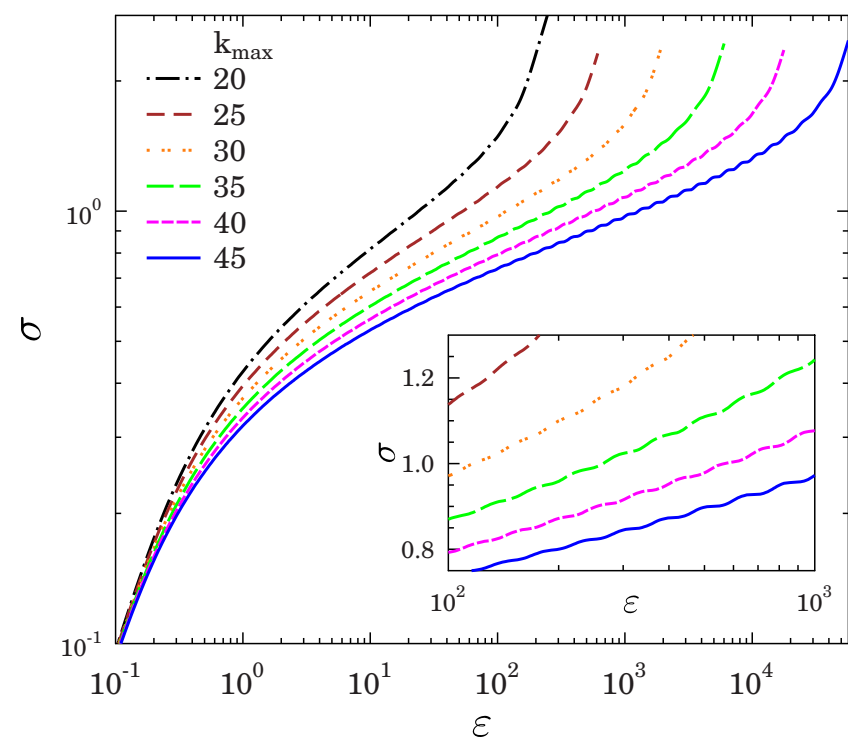

FIG. 8. (Color online) Constitutive curve for the model with sorted failure thresholds and residual stiffness with $\lambda=1, m=2$ for various values of $k_{\max }$ in a double-logarithmic plot, under stress controlled loading. The curves display the same asymptotic behavior as the residual stiffness curves in Fig. 2, although it is not visible in this logarithmic representation. The inset shows a section of the constitutive curves on a logarithmic-linear scale, where the onset of oscillations with increasing $k_{\max }$ is more visible.

$$
m_{k}(\varepsilon)=a^{k} \varepsilon\left[1-G_{(k+1)}\left(a^{k} \varepsilon\right)\right] G_{(k)}\left(a^{k-1} \varepsilon\right),
$$

practically coincide with the observed peaks of avalanches, furthermore, setting $k=k_{\max }$ also yields good estimates for the critical stress and strain; see Fig. 6.

An oscillatory regime also appears if residual stiffness is present, as shown in Fig. 8 for stress controlled simulations for various values of $k_{\max }$, where oscillations are clearly visible for $k_{\max } \geq 30$, as in the case without residual stiffness. Again, the appearance of oscillations depends on the choice of $k_{\max }$, and sets in at $k_{\max } \approx 30$. With increasing $k_{\max }$ also pronounced strain hardening occurs, whereas in the conventional CDFBM a plastic plateau is present. After passing the strain hardening regime, the bundles attain an asymptotic regime of constant slope $a^{k_{\max }}$ for all values of $k_{\max }$, where also macroscopic failure occurs for all cases investigated. It should be noted that therefore at low values of $k_{\max }$, i.e., without oscillations, the slope of the constitutive curve is always finite positive, whereas for higher values of $k_{\max }$, there are sections of the constitutive curve with zero slope due to the oscillations.

\section{B. Bursts of fiber breakings}

The presence of the oscillations, and therefore of a locally vanishing slope, has a distinguished effect on the distribution of avalanche sizes, as demonstrated by Fig. 9 for the case with residual stiffness, and in Fig. 10 for the case without; this is also suggested by the presence of large size avalanches in Fig. 7 (no residual stiffness). It can be seen that in the presence of residual stiffness, Fig. 9, two remarkable 


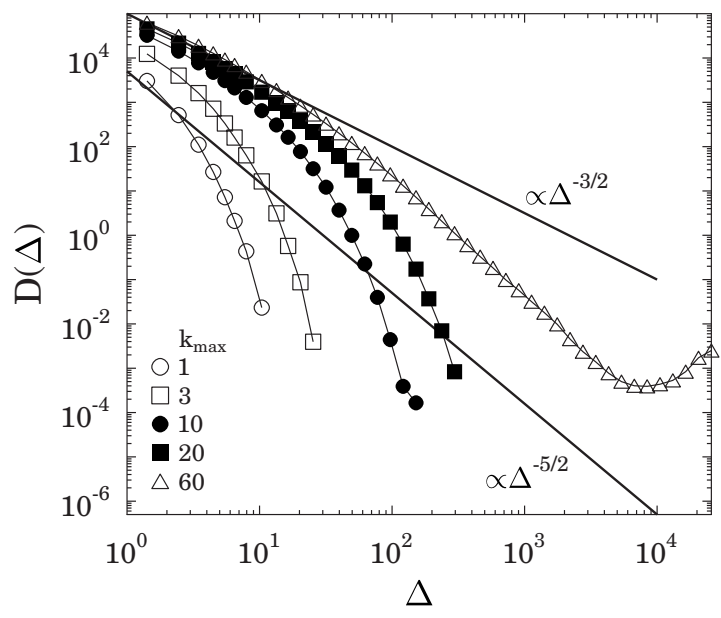

FIG. 9. Avalanche size distributions for the CDFBM with sorted failure thresholds for various values of $k_{\max }, 1 \leq k_{\max } \leq 60$ and residual stiffness. Results from stress controlled simulations of $L$ $=201$ fiber bundles, averaged over 100 realizations.

features are present. First, for all values of $k_{\max }$, there is a regime with a power law of exponent $-3 / 2$ for small avalanche sizes. Second, for small values of $k_{\max }$, an exponential cutoff appears for the larger avalanches; the presence of the initial power law regime with a following cutoff is confirmed by rescaling both axes by the average size of the largest avalanche, $\left\langle\Delta_{\max }\right\rangle$. It is important to note that the distributions $D(\Delta)$ can be collapsed onto a single master curve, which was then fit by the functional form

$$
D(\Delta) \propto\left(\frac{\Delta}{\left\langle\Delta_{\max }\right\rangle}\right)^{-3 / 2} \exp \left(-\frac{\Delta}{c\left\langle\Delta_{\max }\right\rangle}\right),
$$

as can be seen in Fig. 11, where the curves with low values $k_{\max } \leq 20$ have been used. The above arguments are supported by the fact that the power law of exponent $3 / 2$ with an

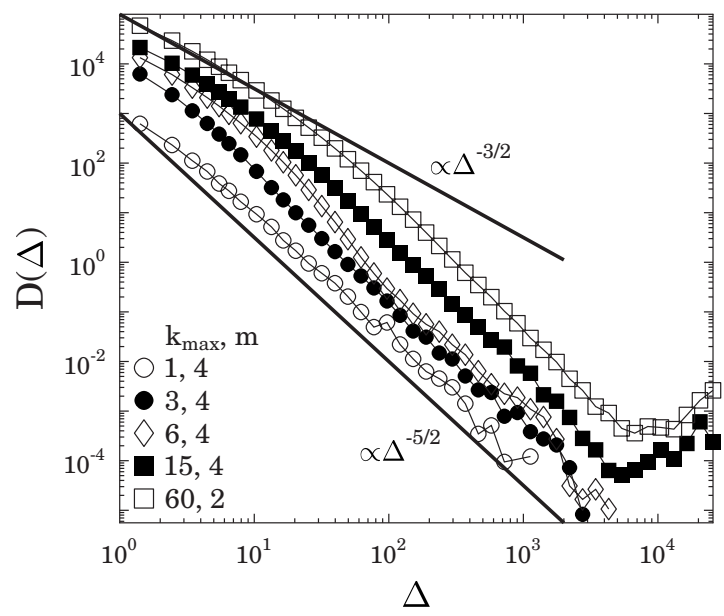

FIG. 10. Avalanche size distributions for the CDFBM with sorted failure thresholds and no residual stiffness for various values of $k_{\max }, 1 \leq k_{\max } \leq 15$, with $m=4$. A comparison to the case of high $k_{\max }=60$ with $m=2$ is presented. Results from stress controlled simulations of $L=201$ fiber bundles, averaged over 200 realizations.

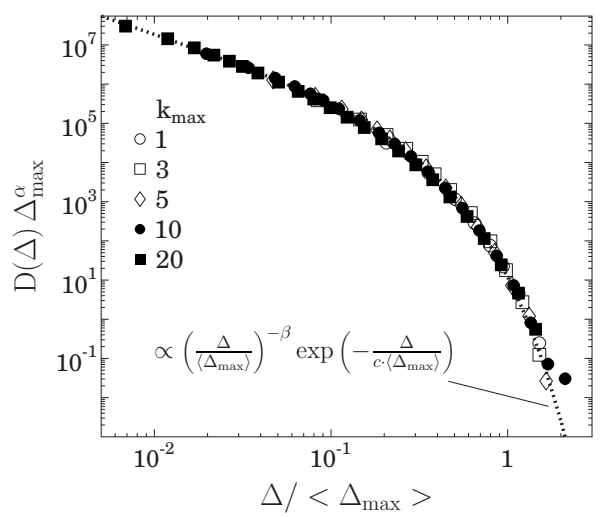

FIG. 11. Rescaled avalanche size distributions for the CDFBM with sorted failure thresholds and residual stiffness, for small values of $k_{\max }$ outside the oscillatory regime. A best fit with the numerical values $\alpha=1.22$ and $\beta=1.5$ for the exponents has been used to obtain the collapse of the curves and the dashed fit curve.

exponential cutoff provides a perfect fit to the master curve obtained numerically. It has to be emphasized that in the fitting, the value of the exponent was fixed to $3 / 2$, and the fit was obtained solely by varying the parameter $c$ in Eq. (16).

For high values of $k_{\max }$, i.e., in the presence of oscillations, a crossover is observed from an initial regime with a power law of exponent $-3 / 2$, to another regime with the mean field power law of exponent $-5 / 2$, and finally to a peaked regime for very large avalanches of about the system size.

A similarly complex behavior is observed in the cases without residual stiffness, see Fig. 10. There, for low values of $k_{\max }$, the usual mean field behavior of a power law with an exponent $-5 / 2$ is observed [22,24]. For intermediate values of $k_{\max }$, a crossover occurs between an initial $-3 / 2$ power law part to a mean field part for larger avalanches, and the position of the crossover shifts to larger avalanche sizes with increasing $k_{\max }$. For higher values of $k_{\max }$, a limiting curve with a crossover at $\Delta \approx 10^{2}$ can be identified, and again a peak of avalanches of the order of the system size is found for $k_{\max }=60$.

The features found in the avalanche size distributions can all be explained on the basis of the fine structure of the oscillations, which is displayed in Fig. 12 for both stress and strain controlled loading, with $k_{\max }=15$ and the choice $m$ $=10$ for the Weibull parameter. From this illustration it becomes apparent that the horizontal plateaus in the stress controlled simulations actually correspond to regions of decreasing stress $\sigma$ under strain controlled loading, which cannot be accessed in the stress controlled mode. Also, for these parameter values, the integral Eq. (11) could be solved analytically by using a computer algebra program, and the analytic solution of Eq. (12) shown in Fig. 12 is in excellent agreement with the simulation results.

In general, a fiber bundle model can only produce large avalanches if the constitutive curve has at least one maximum, where the susceptibility to a small increment of the external force diverges $[12,23]$. Avalanches with a power law distribution are generated in the vicinity of the maximum of $\sigma(\varepsilon)$, where the shape of the maximum determines the value 


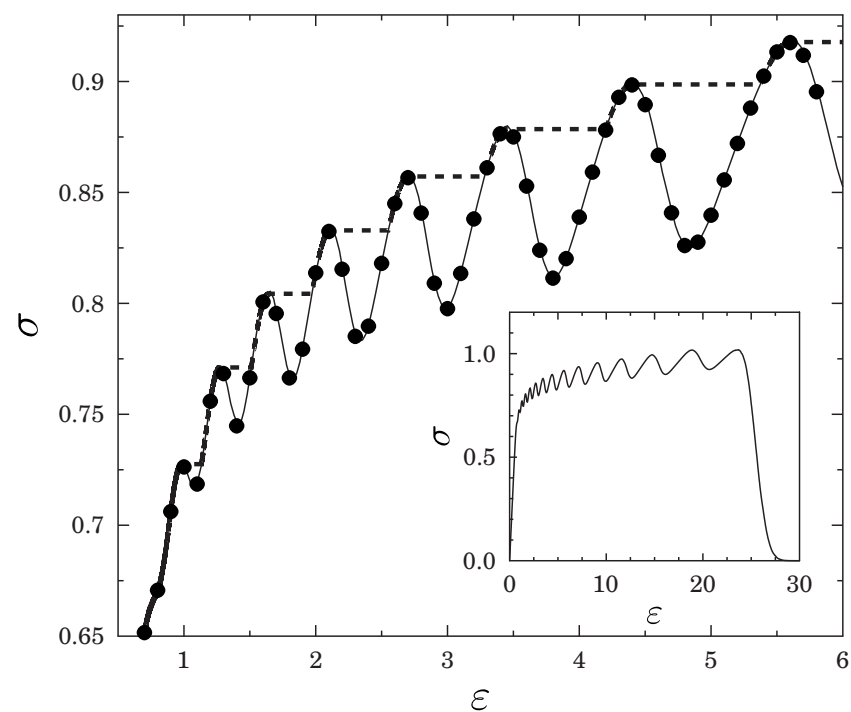

FIG. 12. Section of the constitutive curve for the model with $k_{\max }=15, m=10$ and no residual stiffness. Solid line: strain controlled simulation; dashed line: stress controlled simulation; dots: exact evaluation of the analytical solution, Eq. (12).

of the exponent $\tau$. Quadratic maxima typically result in $\tau$ $=5 / 2$, the value obtained in the absence of both oscillations and a residual stiffness term. If loading is stopped at a strain $\varepsilon_{s}$ before reaching the maximum, i.e., before global failure occurs, an exponential cutoff in the avalanche size distribution appears [13], which is visible in Figs. 9 and 11, where due to the residual stiffness term the bundle fails macroscopically after passing exclusively through regions of finite positive slope, without quadratic maxima, as for the cases $k_{\max } \leq 30$ no oscillations occur.

In the oscillatory regime, however, $\sigma(\varepsilon)$ passes a series of consecutive maxima with an increasing amplitude. Under stress controlled loading the system jumps from a local maximum of $\sigma(\varepsilon)$ to the ascending side of the next maximum which is somewhat higher than the previous one, see Fig. 12. The jump implies that a large amount of fiber breakings occur in a single avalanche removing all fibers which have breaking thresholds lower than the load of the ending point of the jump on the next peak of $\sigma(\varepsilon)$. Consequently, when loading is continued along the ascending side of the peak determined by Eq. (15), the response of the system is determined by a disorder distribution which is critical in the sense of Refs. [32,33], i.e., weak fibers are removed so that the lower cutoff of the disorder distribution falls close to the local critical deformation, the location of the next peak. As it has been shown in $[34,35]$, when the disorder distribution approaches criticality, the avalanche size distribution exhibits a crossover from a power law with an exponent $\tau=3 / 2$ for the small avalanches, to another exponent of $\tau=5 / 2$ for the large ones, irrespective of the effective range of interaction. This effect can be recognized in the $k_{\max }=60$ curve in Fig. 9.

The same argumentation holds for the case without residual stiffness: here, a global quadratic maximum is always present, so for low values of $k_{\max }$ the mean field exponent $-5 / 2$ is found. However, in the presence of oscillatory structures, there appear a series of local quadratic maxima, and

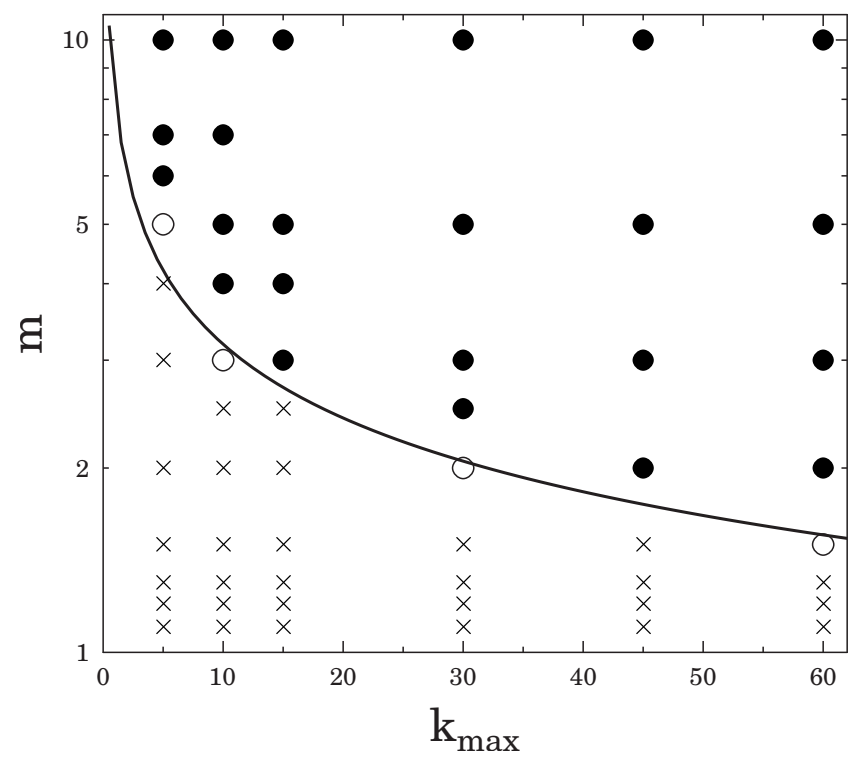

FIG. 13. Phase diagram denoting the appearance of oscillations in the constitutive curves of the model with sorting and no residual stiffness, and the presence of pronounced spikes in the avalanche size distribution. Filled circles: oscillatory regime; crosses: normal regime; unfilled circles: regimes cannot be distinguished. The solid line denotes the approximate location of the separatrix between the two regimes.

the stepping effect described above yields a series of local critical threshold distributions, which results in the crossover of exponents visible in the avalanche size distribution, cf. Fig. 10.

It has to be stressed that the effects found here in the CDFBM with sorting have no equivalent counterparts in the conventional CDFBM without sorting. There, the macroscopic behavior yields a plastic plateau and no steps appear in the constitutive curve; consequently, the size distribution of avalanches shows no signatures of criticality [15]. The existence of oscillations, synchronized avalanche bursts, and a critical crossover of the avalanche size distribution exponents is a genuine peculiarity of the order statistics accompanying sorting.

The analytical argumentation presented above also makes it clear that oscillations cannot appear for all disorder distributions and all values of $k_{\max }$; rather, its appearance is restricted to combinations of either large $k_{\max }$ and high disorder corresponding to low values of the Weibull parameter $m$, or smaller values of $k_{\max }$ and correspondingly low disorder, such that the maxima defined through Eq. (15) are clearly separated. Figure 13 presents a numerical survey of the apparent occurrence of oscillations. One can see that a well defined and smooth separatrix can be found which isolates the regime with oscillations from the regime without in the $\left\{k_{\max }, m\right\}$ parameter space. A word of caution on the physical relevance of these results is in order: for $a=0.8$ and $k_{\max }$ $=60$ the Young modulus at the point of failure is reduced by a factor of $a^{k_{\max }} \approx 10^{-6}$. However, as illustrated in Fig. 13, it turned out that oscillations appear also for considerably lower values of $k_{\max }$, and the Young modulus at failure is accordingly much higher. To name just one example, for $a$ 
$=0.8, \lambda=1.0, m=6$, and $k_{\max }=5, a^{k_{\max }} \approx 0.33$, which compares favorably to experimental situations.

\section{CONCLUSIONS}

Motivated by experimental observations on the fracture process of composite systems having a hierarchy of length scales, we extended the continuous damage fiber bundle model by taking into account that a hierarchy of length and energy scales for damage initiation and crack growth and propagation exists. Since macroscopic damage and arrest event are based on large defects and high strength zones in the material, their number is not a constant but varies between samples of the same production batch. Therefore two additional features have been added to the classical CDFBM, and their effect on the microscopic and the macroscopic damage evolution has been investigated.

First, the maximum number of failures $k_{\max }$ has been modeled as a Poissonian random variable, which incorporates the existence of disorder with respect to the finite number of macroscopic defects and strength zones which can effectively govern the macroscopic breaking of certain materials. The presence of the Poissonian term has a distinct effect on the constitutive behavior as it induces a hardening regime, which becomes more pronounced for small average values of $k_{\max }$. For the microscopic behavior, the introduc- tion of the Poissonian distributed $k_{\max }$ leaves the distribution of avalanche sizes invariant, and a crossover from a power law with an exponent $-5 / 2$ to a power law with another exponent -2.12 for increasing values of $\left\langle k_{\max }\right\rangle$ is found, in analogy to the conventional CDFBM.

In a next step we introduced sorted failure thresholds in the model and explored the inherently complex behavior in this case by analytical and numerical means. A parameter regime has been identified where the damage evolution of all fibers synchronizes and considerable changes to the microscopic quantities can be observed, depending on the amount of disorder and the maximum number of allowed failures. It was shown that the extreme value statistics of failure thresholds has a substantial effect on the fracture process of the system both on the micro and macro level. This theoretical study can be particularly important for composite materials produced by assembling components with a large variation in their respective defects. The application of this model to the failure process of large wooden structures is in progress.

\section{ACKNOWLEDGMENTS}

We are grateful to M. Schrank for experimental contributions and discussions. This work has been supported by the project SFB 381. F.K. acknowledges financial support of the Research Contracts No. OTKA T049209 and No. NKFP-3A/ 043/04.
[1] Statistical Models for the Fracture of Disordered Media, Random Materials and Processes, edited by H. J. Herrmann and S. Roux (Elsevier, Amsterdam, 1990).

[2] Damage and Fracture of Disordered Materials, edited by D. Krajcinovic and J. van Mier (Springer, Berlin, 2000).

[3] F. Kun, S. Zapperi, and H. J. Herrmann, Eur. Phys. J. B 17, 269 (2000).

[4] D. Hull and T. W. Clyne, An Introduction to Composite Materials, Cambridge Solid State Science Series, 2nd ed. (Cambridge University Press, Cambridge, UK, 1996).

[5] S. Thelandersson and H. J. Larsen, Timber Engineering (Wiley, New York, 2003).

[6] A. Guarino, S. Ciliberto, and A. Garcimartin, Europhys. Lett. 47, 456 (1999).

[7] S. Zapperi, H. J. Herrmann, and S. Roux, Eur. Phys. J. B 17, 131 (2000).

[8] W. A. Curtin, Phys. Rev. B 55, 11270 (1997).

[9] Y. Charles, F. Hild, and S. Roux, J. Eng. Mater. Technol. 125, 333 (2003).

[10] S. Roux, D. Vandembroucq, and F. Hild, Eur. J. Mech. A/Solids 22, 743 (2003).

[11] F. Kun, F. Raischel, R. C. Hidalgo, and H. J. Herrmann, Modeling Critical and Catastrophic Phenomena in Geoscience, Lecture Notes in Physics No. 705 (Springer, Berlin, 2006), pp. 57-92.

[12] J. V. Andersen, D. Sornette, and K. T. Leung, Phys. Rev. Lett. 78, 2140 (1997).

[13] M. J. Alava, P. K. V. V. Nukala, and S. Zapperi, Adv. Phys. 55,
349 (2006).

[14] S. R. Pride and R. Toussaint, Physica A 312, 159 (2002).

[15] R. C. Hidalgo, F. Kun, and H. J. Herrmann, Phys. Rev. E 64, 066122 (2001).

[16] S. Zapperi, A. Vespignani, and H. E. Stanley, Nature (London) 388, 658 (1997).

[17] P. Niemz, Physik des Holzes und der Holzwerkstoffe (DRWVerlag, Stuttgart, 1993).

[18] D. Green, R. Tandon, and V. Sglavo, Science 283, 1295 (1999).

[19] A. Hansen and S. Roux, in Damage and Fracture of Disordered Materials, CISM Courses and Lectures No. 410, edited by D. Krajcinovic and J. van Mier (Springer, Wien, 2000), Chap. 2, pp. 17-101.

[20] R. H. Randles and D. A. Wolfe, Introduction to the Theory of Nonparametric Statistics (Wiley \& Sons, New York, 1979).

[21] Bautechnik 83, 667 (2006).

[22] M. Kloster, A. Hansen, and P. C. Hemmer, Phys. Rev. E 56, 2615 (1997).

[23] D. Sornette, J. Phys. A 22, L243 (1989).

[24] A. Hansen and P. C. Hemmer, Phys. Lett. A 184, 394 (1994).

[25] Phani Kumar V. V. Nukala and S. Simunovic, Phys. Rev. E 72, 041919 (2005).

[26] D. L. Turcotte and M. T. Glasscoe, Tectonophysics 383, 71 (2004).

[27] R. C. Hidalgo, C. U. Grosse, F. Kun, H. W. Reinhardt, and H. J. Herrmann, Phys. Rev. Lett. 89, 205501 (2002).

[28] L. Moral, J. B. Gomez, and Y. Moreno, J. Phys. A 34, 9983 
(2001).

[29] L. Moral, Y. Moreno, J. B. Gomez, and A. F. Pacheco, Phys. Rev. E 63, 066106 (2001).

[30] R. Hernandez, D. A. Bender, B. A. Richburg, and K. S. Kline, Wood Fiber Sci. 24, 294 (1992).

[31] U. Divakaran and A. Dutta, Phys. Rev. E 75, 011109 (2007).

[32] S. Pradhan, A. Hansen, and P. C. Hemmer, Phys. Rev. Lett. 95, 125501 (2005).
[33] S. Pradhan and B. K. Chakrabarti, Modeling Critical and Catastrophic Phenomena in Geoscience, Lecture Notes in Physics No. 705 (Springer, Berlin, 2006), pp. 459-477.

[34] S. Pradhan, A. Hansen, and P. C. Hemmer, Phys. Rev. E 74, 016122 (2006).

[35] F. Raischel, F. Kun, and H. J. Herrmann, Phys. Rev. E 74, 035104(R) (2006) 